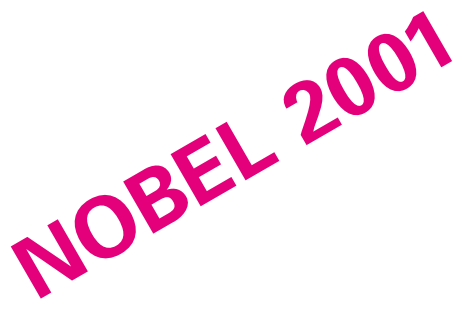
d'être attribué à trois chercheurs du monde de la chimie organique : les Professeurs William S. Knowles (SaintLouis, Missouri, États-Unis) et Ryoji Noyori (Université de Nagoya, Chikusa, Nagoya, Japon) pour leurs travaux sur les réactions d'hydrogénation catalysées "par chiralité" (voir glossaire), et le Professeur K. Barry Sharpless (Institut de Recherche Scripps, La Jolla, Californie, États-Unis), pour ses travaux sur les réactions d'oxydation catalysées «par chiralité". Il est incontestable que ces trois chercheurs ont fait franchir un pas gigantesque à la synthèse organique au cours des dernières vingt années. Leur recherche a porté à la fois sur les concepts, ce qui est considéré comme primordial pour l'attribution du prix Nobel, mais également sur des applications industrielles immédiates, ce qui est beaucoup plus rare.

Certaines réactions chimiques sont largement utilisées en synthèse organique. Il s'agit notamment de la réduction (ou hydrogénation) des alcènes (liaison C-C double) ou des composés carbonylés (cétones), et de l'activation des alcènes par oxydation et de leur transformation en époxydes. Si les réactions d'oxydation et de réduction étaient, dans le passé, assez bien maîtrisées, le contrôle de la chiralité des produits obtenus ne l'était en revanche pas. L'approche des réactifs sur la molécule à transformer se fait en effet de manière égale par les deux faces, et l'on obtient alors un mélange racémique (voir glossaire), c'est-à-dire contenant, à parts égales, deux composés non superposables, les isomères

\section{PRIX NOBEL DE CHIMIE 2001 \\ William S. Knowles, Ryöi Noyori, K. Barry Sharpless}

\section{La catalyse asymétrique}

\author{
André Mann, Camille-Georges Wermuth
}

(figure 1). Or, ces isomères optiques peuvent avoir des activités biologiques différentes et, à partir des années 1960, la demande de substances chirales pour la biologie (notamment de médicaments) devint pressante. Il devenait alors indispensable de produire des médicaments ou des outils biologiques propres au sens premier du terme, c'est-à-dire exempts aussi de leur isomère optique.

On connaissait déjà quelques exemples de réactions d'hydrogénation catalysées par des métaux de transition qui, en présence d'acide tartrique ou de certains autres produits naturels, délivraient en fin de réaction l'un des énantiomères en excès, par une catalyse asymétrique. Cependant, chaque cas était un cas particulier et il n'existait pas à l'époque de concept général. Ce "verrou» a été levé par deux découvertes majeures. La première, celle de Willinkson (Prix Nobel de Chimie en 1973), est la découverte de la catalyse homogène (milieu réactionnel homogène) à l'aide de métaux de transition (Rhodium, Ruthénium...). La seconde, celle de Knowles et Noyori [1-4], est l'utilisation de catalyseurs chiraux. Ceux-ci sont formés de composés phosphorés, appelés phosphines, qui sont complexés aux métaux de transition

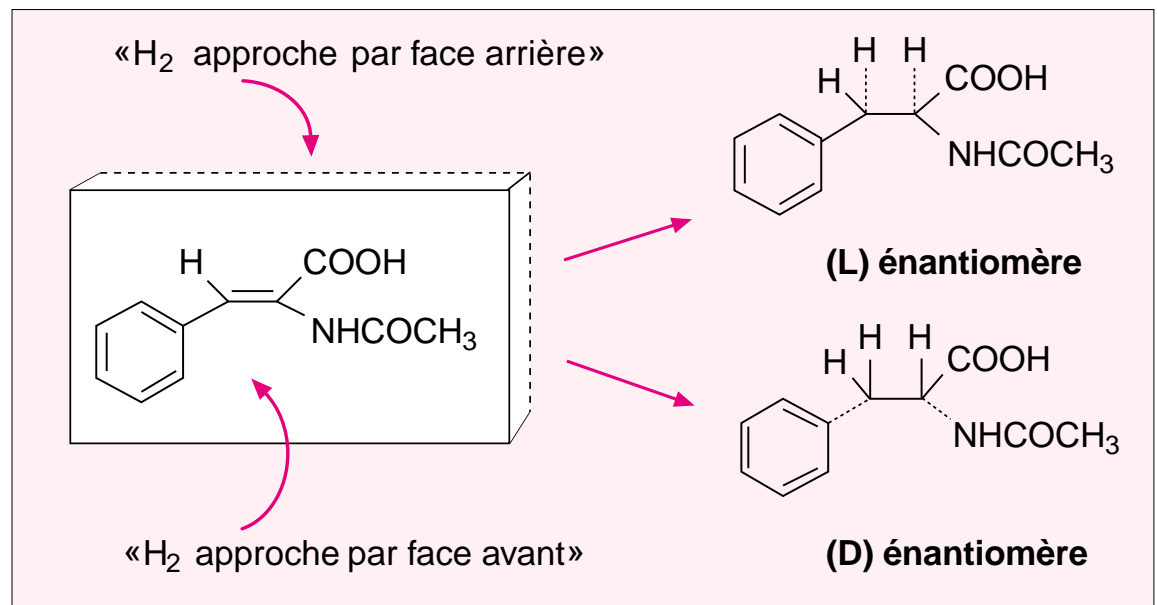

Figure 1. Réaction d'hydrogénation. Au cours de l'hydrogénation de ce précurseur de la phénylalanine, les deux faces de la molécule sont équivalentes pour l'attaque du dihydrogène. Dans les conditions normales, la réaction produit un mélange racémique, c'est-à-dire un mélange à parts égales des deux énantiomères, lévogyre (L) et dextrogyre (D). Si I'on ajoute au milieu d'hydrogénation un catalyseur chiral, le dihydrogène ne pourra approcher qu'une seule face de la molécule et le produit de la réaction sera plus ou moins enrichi pour l'un des énantiomères. Les catalyseurs chiraux sont des métaux complexés à des ligands dont la chiralité détermine celle du produit obtenu.

$m / s n^{\circ} 11$, vol. 17 , novembre 2001 
(figure 2). Ces catalyseurs orientent le transfert d'hydrogène sur une face seulement de la double liaison, ce qui permet de produire de façon majoritaire un seul énantiomère. Les excès énantiomériques (voir glossaire) deviennent alors proches de la perfection.

Ces travaux ont notamment permis de préparer industriellement des composés comme la L-Dopa [(S)-3-(3,4-dihydroxyphenyl)alanine], médicament utilisé dans la maladie de Parkinson, dans des conditions quasi idéales avec un rapport substrat/catalyseur très faible, et la (L)-phénylalanine un composant de l'aspartame, l'édulcorant de synthèse. Si Knowles a poursuivi sa carrière en optimisant les conditions d'hydrogénation, Noyori a développé d'autres aspects de la chimie organique où la catalyse joue également un rôle important. Il a notamment introduit une molécule que l'on peut qualifier de "miraculeuse", le BINAP, bisphosphine à atropisomérie (voir glossaire), un bis-ligand des métaux de transition. Le BINAP, qui est donc accessible sous ses deux formes optiques, permet en effet des réductions d'alcènes et de cétones avec des puretés énantiomériques parfaites. L'apport de Noyori a été déterminant pour la fabrication industrielle de motifs présents dans certains antibiotiques comme les carbapenems, mais aussi pour celle de diverses fragrances comme le menthol.
Les travaux du Professeur Barry Sharpless se situent, eux, dans le domaine de l'oxydation, la réaction d'oxydation d'alcènes qui utilise l'oxyde de ruthénium porte d'ailleurs le nom d'oxydation de Sharpless. Mais c'est l'époxydation d'alcools allyliques qui est la réaction la plus connue du groupe de Sharpless, et l'une des plus utilisées pour introduire la chiralité dans une molécule [5]. Cette réaction est fondée sur un nouveau concept : la réaction est contrôlée par le réactif et non par le substrat. C'est donc le réactif qui va produire la chiralité en s'approchant du substrat par une seule face de la double liaison à oxyder, ce qui s'appelle en jargon chimique un stéréocontrôle.

En 1980, Sharpless a utilisé un mélange de $\mathrm{Ti}(\mathrm{OiPr})_{4}$ (tétra-isopropoxide de titanium) et $\mathrm{tBuOOH}$ (peroxyde de tertio-butanol) comme oxydant, et un dialkyltartrate sous forme d'énantiomère pur, comme source de chiralité, pour oxyder un alcool allylique en son époxyde (figure 3). Les excès énantiomériques obtenus étaient quasi parfaits et la réaction d'un emploi très général. Les quantités de ces composés utilisées pour l'oxydation étaient d'abord proches de celle du substrat, puis des conditions permettant de rendre le processus catalytique ont été trouvées. $\mathrm{Si}$, maintenant, le substrat est un alcool allylique racémique et qu'on le traite avec le mélange oxydant, on obtient ce qu'on appelle une résolution cinétique : un des deux énantiomères de l'alcool allylique va conduire à l'époxyde d'alcool sous forme d'énantiomère pur et le second donnera l'autre énantiomère de l'alcool allylique qui sera aussi pur (figure 3)! Ceci revient à résoudre le mélange racémique de l'alcool allylique de départ par une réaction chimique qui ressemble à une réaction enzymatique. Sharpless a poursuivi alors ses recherches sur deux autres réactions, la di-hydroxylation et l'hydroxyamination asymétriques d'alcènes. Ces deux réactions sont assez générales, elles emploient comme catalyseur chiral un composé à base de quinine et comme oxydant de l'oxide d'osmium.

Dans la communauté des chimistes français, beaucoup sont déçus que le Professeur Henri Kagan n'ait pas été honoré par le Comité Nobel. Sans être cocardier, on peut considérer que Henri Kagan a joué un rôle majeur dans la catalyse asymétrique. Il a en effet été le premier à valider le concept des phosphines comme ligand de métaux, notamment par l'introduction du catalyseur DIOP [6]. De plus, il a montré l'importance de la symétrie axiale C2 du catalyseur (figure 2). En effet, quand Knowles (1968) n'obtenait que des excès énantiomériques de $15 \%$,



Figure 2. Ligands utilisés pour produire des catalyseurs chiraux des réactions d'hydrogénation. Les catalyseurs chiraux sont formés de ligands complexés à des métaux de transition comme le rhodium ou le ruthénium. Les ligands de symétrie axiale C2, le DIOP et le DIPAMP, sont utilisés pour les réactions d'hydogénation. Le ligand BINAP, dont les deux noyaux naphtaléniques sont situés dans des plans perpendiculaires (atropisomérie), est d'un usage quasi universel en catalyse. Ces catalyseurs servent à construire des édifices moléculaires encombrés sur une face du substrat au moyen des fonctions présentes et sur le substrat et le ligand qui complexent le métal.

\section{$+2$

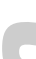

 .}




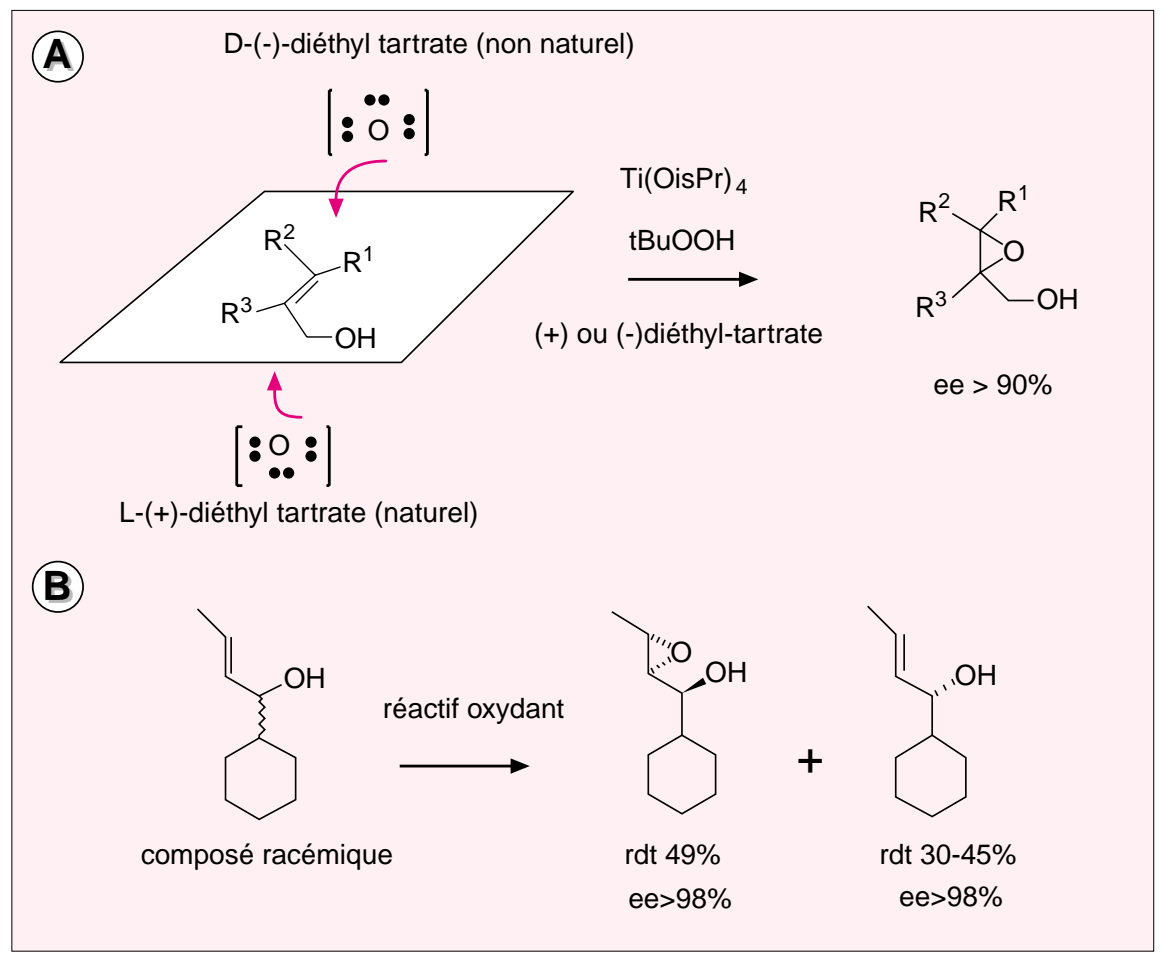

Figure 3. Époxydation de Sharpless. Dans cette réaction, c'est le mélange oxydant à base d'isopropoxyde de titane $\mathrm{Ti}(\mathrm{Ois} \mathrm{Pr})_{4}$ et de tartrate de diéthyle (optiquement pur), ainsi que la présence de la fonction $\mathrm{OH}$ de l'alcool allylique qui sont les éléments déterminants pour produire une bonne induction asymétrique. $\boldsymbol{A}$. La réaction d'oxydation d'un alcool allylique non chiral produit l'époxy-d'alcool sous forme énantiomériquement pure, la chiralité $d u$ tartrate déterminant celle de l'époxy-alcool. B. L'oxydation d'un alcool allylique racémique donne d'une part un énantiomère de l'époxy-alcool, et d'autre part l'autre énantiomère de l'alcool allylique. Les excès énantiométriques de ces réactions sont presque parfaits.

Kagan réalisait pour la même réaction des excès énantiomériques de $80 \%$ (en 1971 !), et a produit les premiers résultats importants dans le domaine de l'hydrogénation catalytique. De plus Henri Kagan a récemment montré que, dans certaines conditions, il n'est même plus besoin de disposer d'un ligand complexant du métal optiquement pur, et qu'il se produit ce qu'on appelle une amplification non linéaire de l'excès énantiomérique. Cette découverte, encore peu exploitée, a permis de mieux comprendre un certain nombre de processus catalytiques. Il permet en outre d'envisager une simplification des procédures de découverte de nouveaux catalyseurs, ce qui constitue l'un des objectifs majeurs de la chimie organique. On peut toutefois signaler qu'il n'y a habituellement qu'au maximum trois lauréats par une exception aurait permis d'éviter cette injustice! Signalons tout de même que le Prix Wolf (2001), qui est une très prestigieuse distinction américaine, a été attribué l'an passé conjointement aux Professeurs Sharpless, Noyori et Kagan. Mais, ne boudons pas notre plaisir, la chimie organique est à l'honneur. Une discipline, la catalyse, a été reconnue comme un domaine de recherche majeur par l'intermédiaire des trois lauréats couronnés cette année. Cette discipline est encore balbutiante, et de nouvelles générations de chimistes auront beaucoup d'efforts à fournir pour la rendre plus prédictive, afin d'améliorer notre santé et notre confort, et peut-être surtout de préserver notre environnement

\section{TIRÉS À PART}

A. Mann.

\section{RÉFÉRENCES}

1. Knowles WS, Sabacky MJ. Catalytic asymmetric hydrogenation employing a soluble, optically active, rhodium catalyst. J Chem Soc Chem Commun 1968 : 1445-6.

2. Knowles WS, Sabacky MJ, Vineyard BDJ. Catalytic asymmetric hydrogenation. Ann $N Y$ Acad Sci 1973 ; 214 : 119-24.

3. Miyashita A, Yasuda A, Takaya H, et al. Synthesis of 2,2'-bis (diphenylphosphino)-1,1'-binapthyl(BINAP) an atropisomeric chiral bis triarylphosphine and its use in the Rh(I)-catalyzed asymmetric hydrogeantion of $\alpha$ (acylamino)acrylic acid. J Am Chem Soc 1980 ; 102 : 7932-4.

4. Noyori R. in "Asymmetric Catalysis in Organic Synthesis " John Wiley and Sons, Inc., New York, 1994.

5. Katsuki T, Sharpless KB. The first pratical method for asymmetric epoxydation. J Am Chem Soc $1980 ; 102$ : 5975-6.

6. Kagan HB, Dang TP. Asymmmetric catalytic reduction with transition metal complexes. I A catalytic system of Rhodium (I) with (-) 2,3- $O$-isopropylidene-2,3-dihydroxy-1,4-bis-(diphenylphosphino)butane, a new chiral diphosphine. J Am Chem Soc 1972; 94: 6429-33.

\section{* GLOSSAIRE *}

1. La chiralité est la propriété qui fait qu'un objet ou un groupe de points ne soit pas superposable avec son image dans le miroir. L'exemple le plus simple de chiralité est celui des mains : l'image de la main gauche dans le miroir est celle de la main droite. En chimie, un atome de carbone lié à quatre substituants différents est l'exemple classique de molécule chirale: il peut exister sous deux formes, de formule chimique identique, non superposables qui sont images l'une de l'autre dans un miroir. Ces deux formes sont des énantiomères.

2. Un mélange racémique contient un mélange à part égale de deux énantiomères. Les énantiomères sont aussi nommés isomères optiques ou antipodes optiques, car ils provoquent une rotation inverse d'une lumière polarisée plane. Donc un mélange racémique à un pouvoir rotatoire nul.

3. L'excès enantiomérique (ee \%) est le rapport de la différence de concentration de l'énantiomère majoritaire et celle du minoritaire divisée par la somme des deux. Pour un ee de $95 \%$ l'un des énantiomères est présent à 97,5 \% dans le mélange et l'autre à $2,5 \%$.

4. L'atropisomérie est la chiralité provenant d'une rotation réduite autour d'une liaison (barrière de rotation > à $20 \mathrm{kcal} /$ mol) qui permet de séparer les énantiomères. Le BINAP en est un parfait exemple.

\section{André Mann}

\section{Camille-Georges Wermuth}

Faculté de pharmacie, Laboratoire de pharmacochimie de la communication cellulaire, UMR 7081, 74, route du Rhin, BP24, F-67401 Illkirch Cedex, France. 

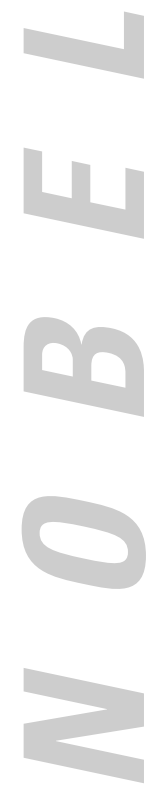